\title{
Energy saving in multi-standard mobile terminals through short-range cooperation
}

\author{
Ayman Radwan ${ }^{*}$ and Jonathan Rodriguez
}

\begin{abstract}
Multi-standard mobile devices are allowing users to enjoy higher data rates and ubiquitous connectivity. These advances are achieved on the expense of higher energy consumption requirements due to the continuous connectivity and the multiple active wireless interfaces. In this article, we use one advantage of the multiple interfaces, namely short-range (SR) communications. Mobile terminals (MTs) use SR cooperative networking to take advantage of the good channel quality of SR links to save energy in multi-standard MTs. In this cooperative network, the combined energy of all MTs is treated as a pool of resources, which is used by all MTs in the network. Towards this end, we propose using cooperation between MTs using SR technology to achieve energy savings. We conduct a quantitative numerical analysis to show the energy saving gains that can be achieved. We derive the energy gains in different use cases, considering different combinations of technologies (WiFi-WiMedia, WiMAX-WiFi and WiFi-WiFi) and different channel conditions. We show that up to $80 \%$ energy savings can be achieved when using a combination of WiMedia as SR and WiFi as long-range technology, compared to using WiFi without cooperation. We also show that SR cooperation can be used to extend the lifetime of the whole network. In such scenario, terminals, which are running out of battery or low in energy, can still relay their data through other MTs, even if this will result in a slight increase in the total consumption of all MTs. This way, source MTs with low battery level benefit from the good condition of the SR channel, extending the lifetime of their batteries. The increase in the energy consumption of the relays can hence be compensated by some kind of payment, which opens opportunities for new business models involving source MTs, relays and network operators.
\end{abstract}

Keywords: energy saving, cognitive radio, cooperation, vertical handover, short-range communications, energy trap

\section{Introduction}

With the development of multi-standard mobile terminals (MTs) and cognitive radio over the past decade, inter-terminal cooperation is now recognized as a feasible solution to control the exponentially increasing trend of energy demand of 4G mobile devices [1-3]. Future Internet services such as High-Quality Video Streaming or Cloud Computing are changing the way MTs, such as laptops and mobile phones, are used since they will be subject to longer periods of connectivity and higher energy consumption. These two factors could have a detrimental effect on the future mobile market, and on the true mobility experience we could enjoy from the so-called "beyond $3 G$ terminals". It is well known that in today's market, a key aspect for the

\footnotetext{
* Correspondence: aradwan@av.it.pt

Instituto de Telecomunicações, Aveiro, Campus Universitário de Santiago, 3800-193 Aveiro, Portugal
}

\section{Springer

C 2012 Radwan and Rodriguez; licensee Springer. This is an Open Access article distributed under the terms of the Creative Commons Attribution License (http://creativecommons.org/licenses/by/2.0), which permits unrestricted use, distribution, and reproduction in any medium, provided the original work is properly cited. mobile consumer is the battery lifetime. Reduced battery life from power hungry terminals means that the market penetration of new future Internet services and applications would be limited. On the other hand, user mobility also plays a vital role in the overall user quality of experience. Future handsets are expected to migrate between different radio technologies in a seamless manner, which means they need to be always connected to the network. In terms of energy consumption, always being connected can also be perceived as always being drained. Therefore, it is realistic to assume that future handsets may lose the "freedom of their mobility" being constrained in proximity to power outlets. In fact, this vision is so recurring that it has widely been labelled as the 4G Trap of MTs [1], and leads to one of the main design challenges of $4 \mathrm{G}$ terminals; on how to make them more energy aware whilst preserving the same transmission bandwidth. Moreover, energy-aware 
terminals also mean that the ICT community is playing a major role in reducing the $\mathrm{CO}_{2}$ emissions emanating from the manufacturing and operation of mobile equipment. In fact, it has been quoted that ICT is responsible for $3 \%$ of the global $\mathrm{CO}_{2}$ emissions, which is equivalent to worldwide $\mathrm{CO}_{2}$ emissions by airplanes or $1 / 4$ of the worldwide $\mathrm{CO}_{2}$ emissions by cars. Therefore, it is important to minimize the energy consumption of future handsets and to make them "green" so that each ICT entity can play their role in making the future ICT landscape more energy conscious.

To address this "green" challenge, many different approaches have been followed to decrease the energy consumption of future mobile devices. A number of efforts have been concentrating on the SLEEP mode, where MTs use synchronous sleep scheduling and trying to increase the periods of SLEEP as much as possible. This approach is followed by the Bluetooth standards (IEEE 802.15.1) [4], also Hibernation mode is suggested in WiMedia, where all network activities are withheld for a number of superframes [5,6]. Another approach is defined in WLAN (IEEE 802.11). Part of the standard is dedicated to describing a feature known as Power Save Mode (PSM) that is available for nodes existing in an infrastructure-based 802.11 WLAN. PSM is based on a synchronous sleep scheduling policy, in which wireless nodes are able to alternate between an active mode and a sleep mode. As a wireless station using PSM first joins an infrastructure-based WLAN, it must notify its access point that it has PSM enabled. The access point then synchronizes with the PSM station allowing it to begin running its synchronous sleep schedule. When packets arrive for each of these PSM stations, the access point buffers them until their active period comes around again. At the beginning of each active period, a beacon message is sent from the access point to each wireless station in order to notify them of these buffered packets. PSM stations then request these packets and they are forwarded from the access point. Once all buffered frames have been received, a PSM station resumes with its sleep schedule wherever it left off. The main drawbacks of PSM mechanism are incapability of providing sufficient QoS for delay sensitive services such as VoIP [7]. The mentioned problem has been addressed by WiFi Alliance in WMM (Wi-Fi Multimedia) Power Save [8].

On the other hand, some research efforts have investigated cooperative communication, but their goal was mainly to enhance the wireless link capacity by taking advantage of the good channel conditions on the shortrange (SR) links and the virtual multiple antennas (virtual MIMO system) [9]. Moreover, some work addressed the energy savings using SR multiple hop relaying. In $[10,11]$, the authors investigated the energy savings using SR multi-hop communications instead of long-range (LR) legacy systems. The authors compared using WLAN in the ad hoc mode to transfer data between source and destination to communicating using an overlay cellular network. The authors show that energy consumption can greatly be reduced using multihop SR communications. The authors also provide some limits on the number of relays based on achieving energy savings and staying below the delay limits of the cellular overlay networks. The study in $[10,11]$ only considers one technology at a time. The mobile device either uses the overlay cellular network or the SR ad hoc WLAN. The results can be considered as good motivations to continue working on SR communications. The authors assumed that it is possible to find a route from sender to receiver using multi-hop relaying. In cellular networks, it is usually hard to find a route between two communicating users. Communicating users are usually too far from each other with many kilometres between them, rendering it hard to establish a routing path from sender to receiver. In this study, we realized that and hence combined the SR multi-hop communications with the LR technology to reduce the energy required to reach the access point or the base station. Other efforts have been devoted to propose new MAC protocols for cooperative SR communication, considered energy efficiency as one of their goals $[12,13]$.

In this study, we consider a new technology, cooperation between mobile devices, which have previously been employed for enhancing single transmission quality. Cooperation will be exploited as a means of establishing an energy-efficient communication path by using the available resources of neighbouring nodes. In fact, the key scenario will adopt an SR communication network as a proxy for establishing LR communication path with the mobile network. Moreover, our solution will also exploit cognitive features in a broad sense since terminals will need to be intelligent in nature. This means that terminals will need to continuously sense, make decisions and react towards their environment in order to preserve power.

In this article, we provide a quantification of the energy savings that can be achieved when using cooperation between MTs to reduce the energy consumption of the MTs, while guaranteeing the provision of the minimum required quality of service. Cooperation takes advantage of the good channel conditions of the SR links, hence achieving higher data rates, at lower energy requirements. We present a detailed energy analysis of the proposed solution and compare with the non-cooperative scenario, to highlight the potential gains achieved through our proposed cooperation solutions. We show the numerical results based on the conducted energy analysis, which explain the energy savings achieved 
through cooperation. The numerical analysis shed light on different use cases, compromising different combinations of SR and LR technologies, including WiFi-WiMedia, WiFi-WiFi and WiMAX-WiFi combinations. The results show a great potential of energy saving reaching $80 \%$ in some cases, while achieving more than $50 \%$ energy savings in all cases. The results also show the maximum number of relays that can be used to achieve energy savings.

The rest of the article is organized as follows. In Section 2, we present the system model and the considered scenarios. Section 3 presents the numerical analysis of the energy consumption of the MTs in both cases, i.e. with and without cooperation. In Section 4, the numerical results are shown, highlighting the different usecases considered for implementation. Section 5 concludes the article and presents some materials for future study.

\section{System model and scenario description}

The system, we consider in our analysis, consists of MTs equipped with multiple interfaces. Each MT has at least one LR technology to communicate directly with an access point/base station and one SR technology to be used in relaying between mobile devices in the cooperation scenario. Some MTs can have more than two interfaces. In our analysis, we use LR technology to describe the technology of the available access point. This is the technology used by MTs to communicate directly with the access point. It is referred to as LR, independent of the type of technology and its coverage ranges. The technology used in cooperation and communication between MTs is considered the SR in our analysis, again independent on the technology used.
The basic scenario is shown in Figure 1. An MT is connected to an LR BS. The MT is almost at the edge of the cell of the BS, implying that the MT is getting the lowest possible QoS offered by the technology with the highest energy requirement, but the signal strength did not drop below the HO threshold; hence, the MT is not yet searching for better signal strength. The MT can also be indoors or behind some solid obstacles, which decreases the QoS even if the MT is not too far from the access point.

The MT is equipped with at least one other interface, an SR interface. We will call this technology SR1. The MT is in the communication range of the SR1 interfaces of other MTs. In the baseline scenario, the MTs do not have the capabilities of using their SR interfaces to form a cooperative cluster and use SR communication to relay data for each other.

For the ease of following the discussion and the derivation, we are going to have some assumptions. These assumptions are just to make the derivation easy to follow. The assumptions can easily be modified to represent more generalized cases. We concentrate on uplink scenario where all source MTs have data of the same size $L$ to transmit. They all transmit using the same packet length.

\subsection{The cooperation scenario}

In the cooperation scenario, MTs take advantage of the energy efficiency of the SR communications. The MTs exploit the SR communication capabilities to reach the LR AP consuming less energy.

To start, the MTs form a cooperative cluster. MTs, with data to transmit, send their data to the cluster head using the SR communications. The cluster head

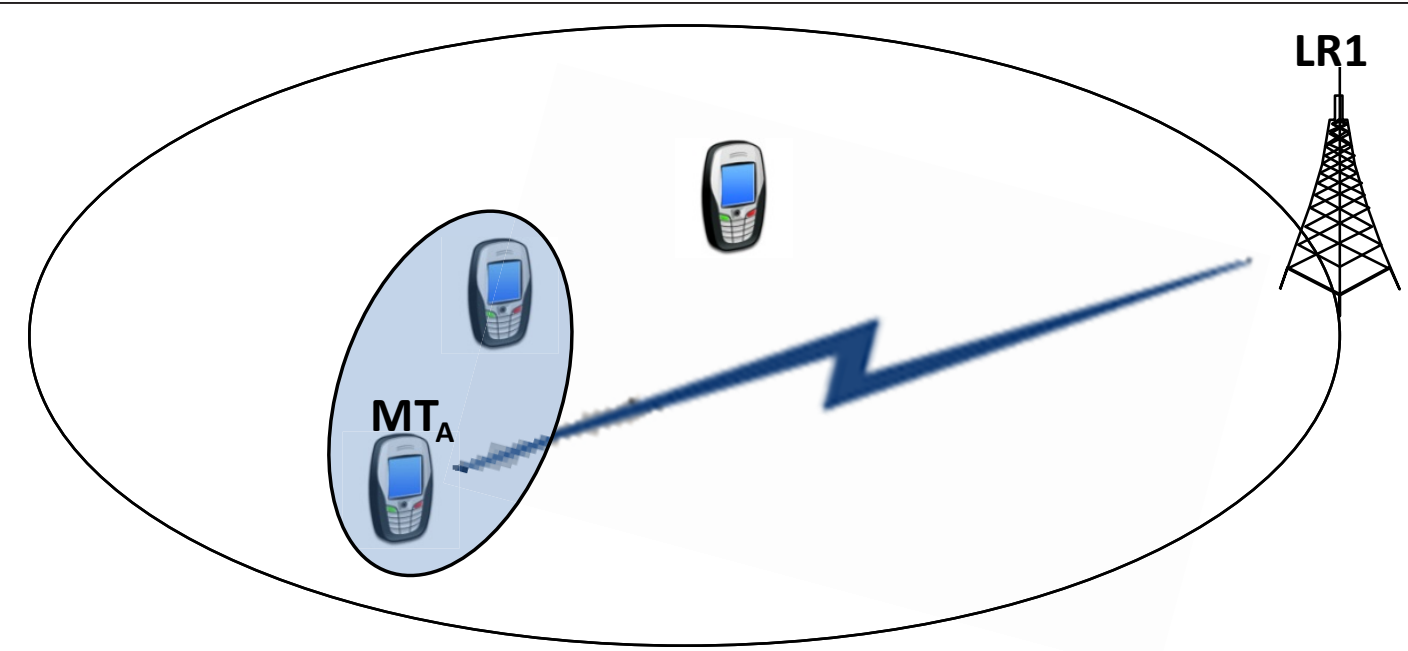

Figure 1 Baseline scenario All MTs are equipped with multiple interfaces. 
then sends the aggregated data from all MTs to the BS using the LR1 communication. Figure 2 shows the proposed cooperative solution scenario, with a cooperative cluster formed of only two MTs (a cluster member and the cluster head).

In the figure, $\mathrm{MT}_{\mathrm{A}}$ has data to send on the uplink towards the BS. The channel between $\mathrm{MT}_{\mathrm{A}}$ and LR1 BS is experiencing bad conditions. MTs can determine that channel conditions are bad, based on the data rates that can be achieved on the link. This can be due to the fact that $\mathrm{MT}_{\mathrm{A}}$ is too far from LR1 BS or that $\mathrm{MT}_{\mathrm{A}}$ is behind a building or some metal blocks, which renders the channel bad. Due to the bad conditions of the direct channel to LR1 BS, $\mathrm{MT}_{\mathrm{A}}$ searches for more energy-efficient connection to upload its data to the network. $\mathrm{MT}_{\mathrm{A}}$ may as well be just running on low battery and the user of $\mathrm{MT}_{\mathrm{A}}$ is willing to spend some money (credits) to save its battery power. At the same time, $\mathrm{MT}_{\mathrm{B}}$ can be willing to sacrifice some of its energy to gain some credits. Moreover, $\mathrm{MT}_{\mathrm{B}}$ can have high battery level or can be connected to a power supply, which means $\mathrm{MT}_{\mathrm{B}}$ is not so concerned with energy consumption.

Using the available context information, $\mathrm{MT}_{\mathrm{A}}$ is able to find out about $\mathrm{MT}_{\mathrm{B}}$ in its vicinity. $\mathrm{MT}_{\mathrm{A}}$ and $\mathrm{MT}_{\mathrm{B}}$ then perform cluster formation, and after negotiation decide that $\mathrm{MT}_{\mathrm{B}}$ is the cluster head and will relay data from $\mathrm{MT}_{\mathrm{A}}$ towards LR1 BS, as shown in Figure 2.

It is worth noting that the connection from $\mathrm{MT}_{\mathrm{A}}$ to $\mathrm{MT}_{\mathrm{B}}$ is using $\mathrm{SR}$ communication, while the connection from $\mathrm{MT}_{\mathrm{B}}$ to LR1 BS is using LR communication.

The described scenario is the simplest one, which is used to show the expected energy savings compared to the baseline scenario. This scenario can be further generalized and become more complicated, by adding more
MTs to the cooperative cluster. The cluster head can also opt to use multiple hops to reach the LR1 BS, instead of sending directly to the BS. The cluster head decides to use more than one hop, in the case when the direct channel between the cluster head and the BS is bad (meaning that the cluster head needs high energy consumption to transmit data using the LR communications the LR1 BS) and the cluster head knows - from the context information and energy efficient routing algorithm - about the availability of a more energy-efficient route towards the BS using multiple hops.

The general scenario is shown in Figure 3. In the figure, there are $N+1$ MTs constituting the cooperative cluster ( 1 cluster head and $N$ cluster members). All $N$ cluster members send data to the cluster head using SR communication. There are $K$ relays between the cluster head and LR1 BS, yielding $(k+1)$ hops on the route. All hops between different relays use SR communications, while the final hop from the last relay to the LR1 BS is using LR link. Using multiple hops may consume more energy in the relaying process, but can still be more energy efficient if the last hop has a good LR link to the BS.

The energy consumption is in general technology dependent; hence, different values of energy savings can be achieved depending on the used technology and the channel conditions which determine the achieved data rates. Different use cases are shown in Section 4 to elaborate on this fact, while highlighting the optimum use cases which yield the most energy savings.

\section{Analysis of energy consumption}

This section will address the analysis of energy consumption and the derivation of equation to quantify the

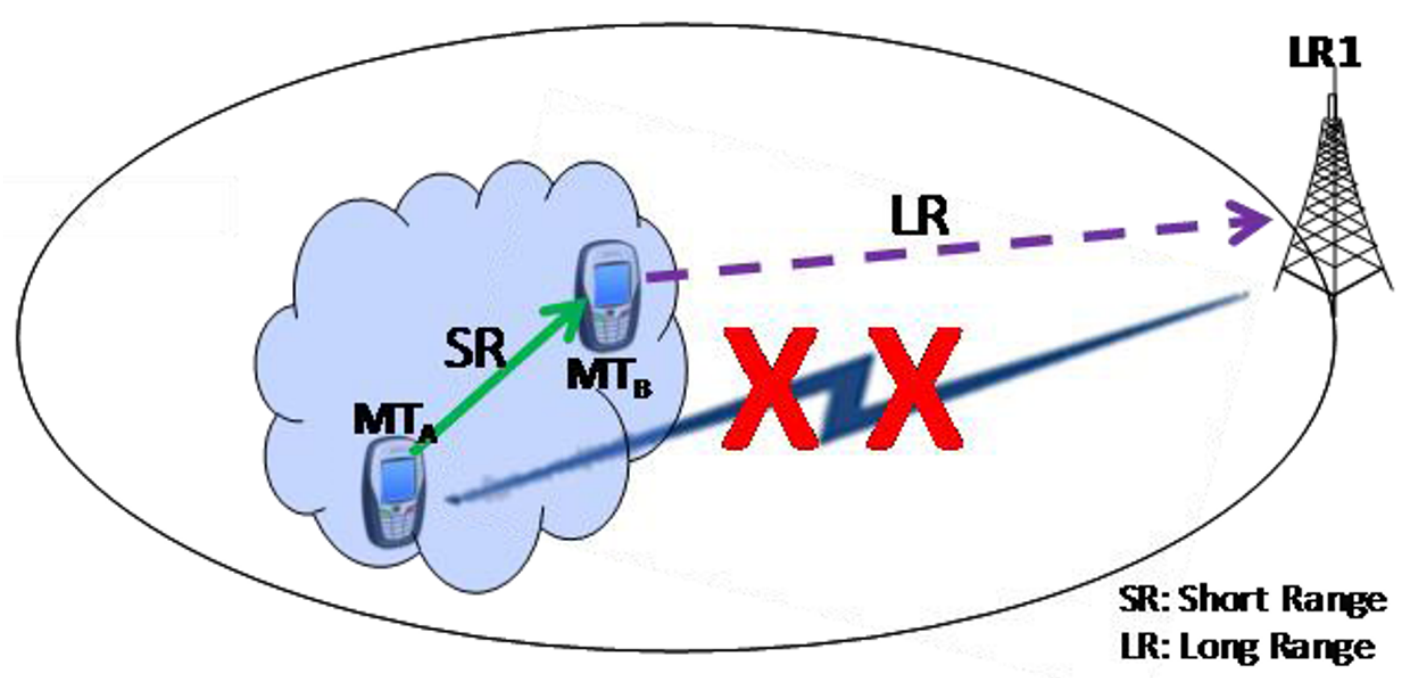

Figure 2 Energy saving using cooperative clusters in homogeneous networks. 


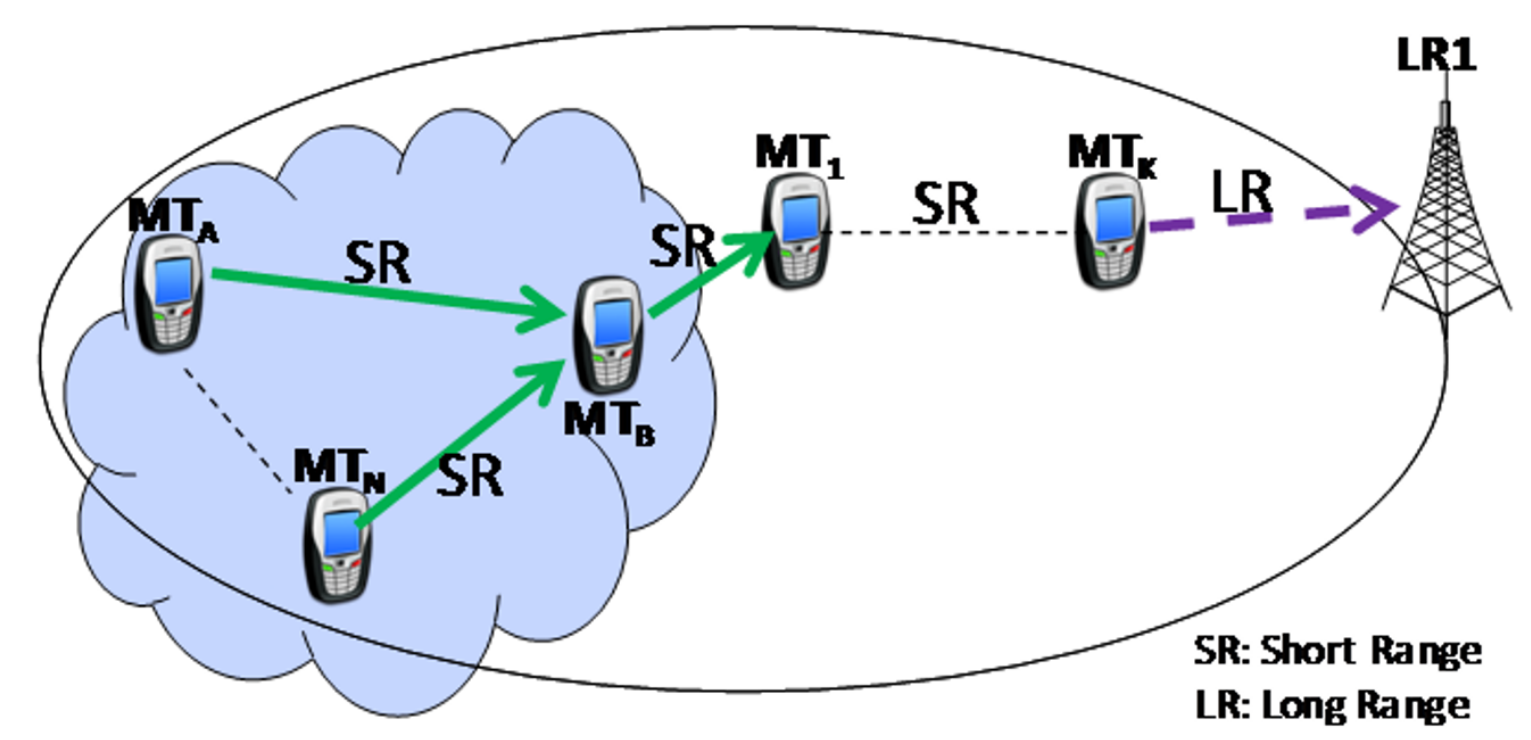

Figure 3 The general case of energy saving using cooperation.

energy gains of using cooperation among MTs. The section is divided into two subsections. The first subsection covers the analysis of energy consumption in the nocooperation case, while the latter discusses the energy consumption when cooperation is used. The second case consists of three stages, which are defined and the energy consumed during each stage is derived.

\subsection{Energy consumption in baseline scenario (no cooperation)}

The energy consumption in the baseline scenario will act as a reference line for comparison with the performance of the proposed cooperative scenarios. The comparison between the performance of the proposed solutions and the performance of the baseline scenario will show the targeted performance enhancement using cooperation.

In the baseline scenario, energy consumption is due to the transmission of data through the LR interface only. During the whole transmission session, only one interface is active transmitting data, while we assume the other interfaces are in the sleep mode.

Based on the discussion above, the consumed energy during the transmission of data in the baseline scenario can be calculated by

$$
E_{\text {NoCoop }}=T_{t x L R 1} P_{T x L R 1}=P_{T x L R 1} L / R_{L R 1}
$$

where $E_{\text {NoCoop }}$ indicates the energy consumed when no cooperation is used. $P_{T x L R 1}$ is the power consumed while in the transmission state of the LR1 interface on the MT, $T_{t x L R 1}$ is the time needed to transmit the data using LR1 interface, $L$ is the length of the transmitted data and $R_{L R 1}$ is the data rate achieved on the LR1 interface.

The amount of consumed energy calculated by (1) only considers the power dissipation due to data transmission by the active interface. Hence, using (1), it can be assumed that all other interfaces are switched off by the user. This is usually not the case in current smart phones. Other interfaces are usually $\mathrm{ON}$ and would be in the sleep state. Taking this into consideration, the total consumed energy is modified to

$$
E_{\text {NoCoop }}=T_{t x L R 1}\left(P_{T x L R 1}+\sum_{j=1}^{M-1} P_{S l j}\right)=\left(P_{T x L R 1}+\sum_{j=1}^{M-1} P_{S l j}\right) L / R_{L R 1}
$$

where $P_{S l j}$ is the power dissipation of interface $j$ during sleep state and $M$ is the number of interfaces equipped on the MT. The summation in the equation represents the sum of power dissipation during sleep state in all other inactive interfaces.

The energy consumption values in the baseline scenario are technology dependent. In Section 4, we will show different values of consumed energy depending on the used technologies for different use cases. We will also show that the achieved reduction in energy consumption is dependent on the types of technologies involved (switched between) in the use cases.

\subsection{Energy consumption in SR cooperative networks}

Using SR cooperation, the total energy consumption consists of the consumed energy on the SR and the LR links, in addition to the overheads resulting from cooperation. Overheads can include node discovery, context 
aware functionalities (context gathering, maintenance and distribution), cluster functionalities (cluster formation, cluster maintenance, etc.). Even with all the cooperation overheads, exploiting SR cooperative clustering still yields more energy-efficient communication, as will be shown in Section 4.

In the beginning, we calculate the energy consumption for the general case, then we are going to modify the derivation to represent certain cases.

For the ease of understanding the calculation, we are going to have some assumptions. These assumptions are just to make the derivation easy to follow. The assumptions can easily be modified to represent more generalized cases. In the calculation, it is assumed that all $N$ cluster members (not including the cluster head) have data of the same size $L$ to transmit. We assume that all cluster members use the same data rate; which is the lowest data rate achieved between cluster members and the cluster head. This assumption makes the results lower bound for the energy savings that can be achieved in a certain use case.

The transmission of data from the $N$ cluster members to the LR1 BS consists of three stages. During the first stage, each cluster member sends its data over an SR link to the cluster head. During the second stage, the cluster head has to transmit the aggregated data from all cluster members to the last relay. This stage compromises all the hops to reach the last relay. The third and last stage represents the last hop from the last relay to the LR1 BS. In this hop, data are transmitted over an LR1 link. We will derive the energy consumption during each stage separately. The total energy consumption to transmit all the data from all $N$ cluster members all the way to the AP is the summation of consumed energy in all three stages.

During the first stage, each cluster member sends its data over an SR link to the cluster head. The energy consumed during the first stage can be expressed as

$$
E_{s t 1}=N\left(P_{T x S R}+P_{R x S R}+(N-1) P_{\mathrm{SISR}}\right) L / R_{\mathrm{SR}}
$$

where $P_{T x S R}, P_{R x S R}$ and $P_{\text {SISR }}$ are the power dissipation during the transmission state of one cluster member, the receiving state of the cluster head, and the sleep state of the rest of the cluster members, respectively, for the SR interfaces. At any point of time during this stage, one cluster member is transmitting to the cluster head, while all other cluster members are in their sleep state. The term $(N-1) P_{\text {SISR }}$ represents the energy consumed during sleep state by all members of the cluster other than the active one transmitting data to the cluster head at the moment. The term $R_{\mathrm{SR}}$ represents the data rate achieved on the SR links, which is the lowest data rate achieved between any cluster member and the cluster head, as explained earlier in the assumptions.

During the second stage, the cluster head has to transmit the aggregated data from all cluster members to the LR1 BS. This part compromises the energy consumed by the $K$ SR hops to reach the last relay, which can be written as follows

$$
E_{s t 2}=K\left[\left(P_{T x S R}+P_{R x S R}\right)+(N+1-1 / K) P_{\text {SISR }}\right] N L / R_{\mathrm{SR}}
$$

where $K$ is the number of relay nodes. In (4), the total length of the aggregated transmitted data is $N L$, consisting of a block of length $L$ from each cluster member. During the relay of data from the cluster head to the LR1 BS, it is assumed that all cluster members switch to sleep mode, since they have finished their data transmission. The energy consumed in the sleep mode of the cluster members is expressed in the second term of (4). The summation reflects the idea that the $N$ cluster members are in the sleep mode during the $K$ hops from cluster head to last relay, while the cluster head is in active mode transmitting during the first hop, then switches to sleep mode during the rest of the hops.

The third and last stage represents the last hop from the last relay to the LR1 BS. In this hop, data are transmitted over an LR1 link. The energy consumed during the transmission of data from the last relay to the LR1 $\mathrm{BS}$ is

$$
E_{s t 3}=\left(P_{T x L R 1}+(N+1) P_{\mathrm{SISR}}\right) N L / R_{\mathrm{LR} 1}
$$

Again, all nodes of the cluster are assumed in sleep mode. It can be seen that in (5), only energy consumption in the transmission state is considered, since the receiving procedure is done on the BS side. In this analysis, we are only comparing the effect of the proposed SR cooperation solution on the energy consumption of the MTs, hence not including the power dissipated by the BS while receiving. In both cases using cooperation or not, the BS dissipates power for receiving the data from MTs. Using cooperation, the achieved data rate on the final LR hop to the BS is at least the same as the case when the MT is sending directly to the BS. The energy consumed by the BS for receiving the data is at most the same as without cooperation. Hence, in addition to saving energy on the MT side, our cooperative solution keeps the energy consumption the same on the BS side if not decreasing it.

For fair comparison with the baseline scenario, we consider the energy dissipated through the LR interface during the whole transmission procedure, assuming that the interface is switched to the sleep mode. The total 
energy consumed through LR1 interface during the cooperative communication to the BS is expressed as

$$
E_{\text {LR1Sleep }}=\left(N L / R_{\mathrm{SR}}+K N L / R_{\mathrm{SR}}+N L / R_{\mathrm{LR} 1}\right)(N+1) P_{\mathrm{SILR} 1}(6)
$$

Equation (6) gives the energy dissipated through LR1 interface of each of the $N+1$ members of the cluster during the whole duration of the three stages.

The total energy consumed for the transmission of $N$ blocks of data of length $L$ from $N$ nodes using cooperative communication to the BS is simply the sum of the energy consumed in the three stages in addition to the energy dissipation through the LR1 interfaces during the three stages. The total energy consumed in the transmission, when cooperation is used, is

$$
E_{\text {Coop }}=E_{s t 1}+E_{s t 2}+E_{s t 3}+E_{\text {LR1Sleep }}
$$

Equation (7) represents the energy consumption in the general case of cooperation. If we only consider one MT transmitting data to the $\mathrm{BS}$, we substitute $N=1$ in the above equations. Moreover, if we assume that the cluster head is transmitting data directly to the LR1 BS using LR communication, $K$ is substituted by zero. For this special case, the energy consumed is calculated by

$E_{\mathrm{Coop}}=\left(P_{T x S R}+P_{R x S R}+2 P_{P S L R 1}\right) L / R_{\mathrm{SR}}+\left(P_{T x L R 1}+2 P_{\mathrm{SISR}}+P_{\mathrm{SLLR} 1}\right) L / R_{\mathrm{LR} 1}$

given that there is one node transmitting and the cluster head is sending data directly to LR1 BS.

For cooperative transmission to be more energy efficient, the value of Equation (8) has to be less than the value of Equation (2) for the case with one MT sending with the cluster head transmitting directly to the LR1 $\mathrm{BS}$, yielding the following condition

$$
\left(P_{T X S R}+P_{R x S R}+2 \text { PSLLR1 }\right) L / R_{\mathrm{SR}}+\left(P_{T X L R 1 \beta}+2 P_{\mathrm{SISR}}+P_{\mathrm{SLLR1}}\right) L / R_{L R 1 \perp B}<\left(P_{T X L R 1 \mathcal{A}}+P_{\mathrm{SISR}}\right) L / R_{L R 1 \mathcal{A}}
$$

In (9), it can be noticed that $P_{T x L R 1}$ has been modified to $P_{T x L R 1_{-} A}$ and $P_{T x L R 1_{-} B}$, while $R_{L R 1}$ has been modified to $R_{L R 1_{-} A}$ and $R_{L R 1_{-} B}$ to imply the possible different power requirements or different achieved data rates on the LR1 interface of different MTs, based on their position with respect to the LR1 AP. This differentiation is very important, and it constitutes the main factor in achieving energy savings. From the equations, it can be seen that the savings in energy consumption depends mainly on the achieved data rates on different channels and the power consumption based on the active interfaces. The idea is mainly to use the SR communications-which is more energy efficient-to reach a MT with a good LR channel to the AP. By doing so, the total transmission time is reduced, hence increasing the sleep time of nodes. This results in decreasing the total energy consumption. As mentioned above, a slight increase in the total energy can be acceptable to save a certain MT from running out of battery.

In Section 4, we show different use cases with different technologies and different channel conditions.

\section{Numerical results}

In order to provide illustrative measures, we compare the energy consumption using cooperative communication between MTs on SR links to the energy consumption without cooperation (i.e. using direct LR to BS/AP). In this section, we are going to show three different use cases, which are different based on the considered LR and SR interfaces on the MTs and available LR network coverage. The three cases are as follows:
A. WiFi (802.11g) as LR and WiMedia as SR
B. WiFi $(802.11 \mathrm{~g})$ as LR and WiFi $(802.11 \mathrm{~g})$ as SR
C. WiMAX as LR and WiFi (802.11g) as SR

In the numerical results, we only compare the energy consumed on the two considered interfaces. This should be a fair comparison between the proposed cooperative solution and the baseline scenario. In our energy calculation, we divide the consumed energy by the length of the transmitted data, to obtain the energy consumed per bit (J/bit) as a fair and easy to reproduce comparison.

The consumed energy within each scenario depends mainly on the power consumption in different states (transmission, receiving, idle or sleep) and how long MTs spend in each state. In all technology interfaces, the sleep state is the lowest power dissipation state, usually with a big gap between the sleep state and all the other states. In our cooperation scenario, the main idea is to achieve high data rates on low-energy-consuming interfaces. This leads to short transmission time (active periods); hence, MTs can be in sleep state for longer periods of time, saving more energy.

As a quick comparison between the three considered technologies, we present the power dissipation in each state for the three technologies listed in Table 1. From the table, the low power dissipation in the sleep state is so obvious compared to other states. Moreover, it can be noticed that WiMedia power requirement is much lower than WiFi or WiMAX, with even higher data rates, but the transmission range is much smaller.

To begin with, Figure 4 shows the energy per bit values for the baseline scenario (No cooperation) for the two considered LR technologies (WiMAX and WiFi). It can be clearly seen the effect of achieved data rates on the energy consumption per bit. Whenever a higher data rate is achieved, less energy is consumed per bit. In the figure, we also show the effect of the energy 
Table 1 Power consumptions of different considered technologies activity states

\begin{tabular}{llll}
\hline Interface type & Power consumption in TX mode $(\mathbf{m W})$ & Power consumption in RX mode $(\mathbf{m W})$ & Power consumption in sleep mode (mW) \\
\hline WiMedia [14] & 350 & 400 & 0.25 \\
WiFi 802.11g [14] & 1900 & 1340 & 75 \\
WiMAX [15] & 1500 & 1500 & 5 \\
\hline
\end{tabular}

consumed on the second interface (SR interface), which is considered in sleep mode since the MT is transmitting on the LR interface. It is clear from the figure that the effect of the consumed energy on the second interface is almost negligible. One of the features of our proposed solution is to keep inactive interfaces in sleep mode.

\subsection{WiFi (802.11g) as LR and WiMedia as SR}

The first use case is using WiMedia (UWB) as the SR technology used in the communication between MTs. The LR technology is assumed to be WLAN IEEE $802.11 \mathrm{~g}$. The power consumption of these technologies in the different modes is listed in Table 1. It can be observed that the power consumption of WiMedia is much lower than that of WiFi. WiMedia can also achieve higher data rates compared to IEEE802.11g; hence, using WiMedia for cooperation can save energy.
In the first set of results, we consider a scenario as the one shown in Figure 2, where there is one device $\mathrm{MT}_{\mathrm{A}}$ transmitting data to the LR AP (WiFi AP). The direct link between $\mathrm{MT}_{\mathrm{A}}$ and the WLAN AP is experiencing bad conditions, resulting in low data rate. $\mathrm{MT}_{\mathrm{A}}$ knows about $\mathrm{MT}_{\mathrm{B}}$ in the vicinity through the context information. $\mathrm{MT}_{\mathrm{A}}$ decides to cooperate with $\mathrm{MT}_{\mathrm{B}}$ to achieve better energy efficiency by relaying its data on the SR through $\mathrm{MT}_{\mathrm{B}} \cdot \mathrm{MT}_{\mathrm{B}}$ transmits $\mathrm{MT}_{\mathrm{A}}$ data over $\mathrm{LR}$ to the WLAN AP. The achieved saving in the energy consumption depends on the achieved data rates on the SR and the LR links in the cooperation scenario. The possible achieved energy efficiency (consumed energy per bit) is plotted in Figure 5.

The figure shows the consumed energy per bit versus the achieved data rate on the SR link. Clearly, the energy efficiency improves with the increase in the achieved data rate on the SR link. The figure shows

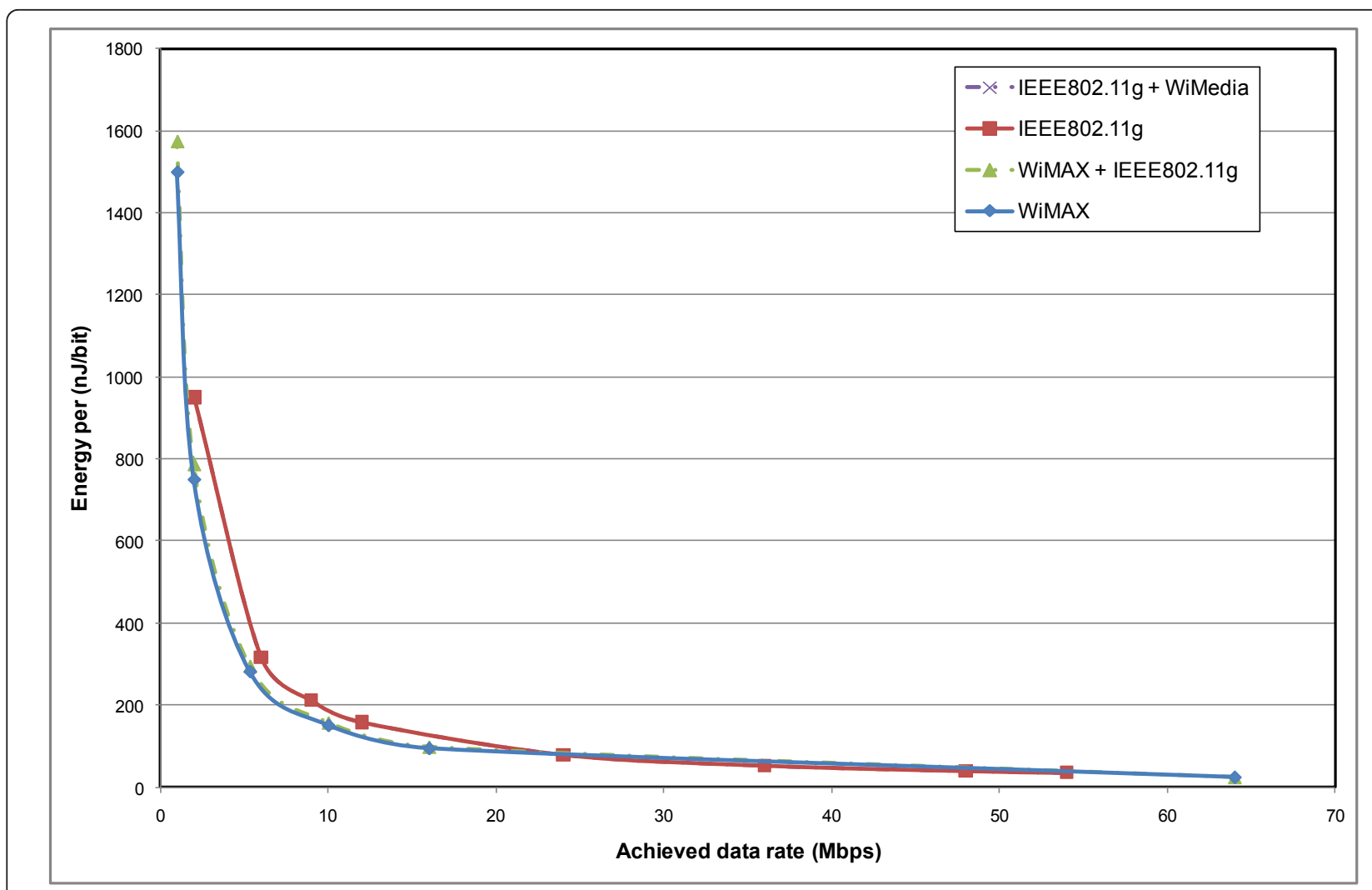

Figure 4 The energy efficiency for different technologies based on achieved data rates. 


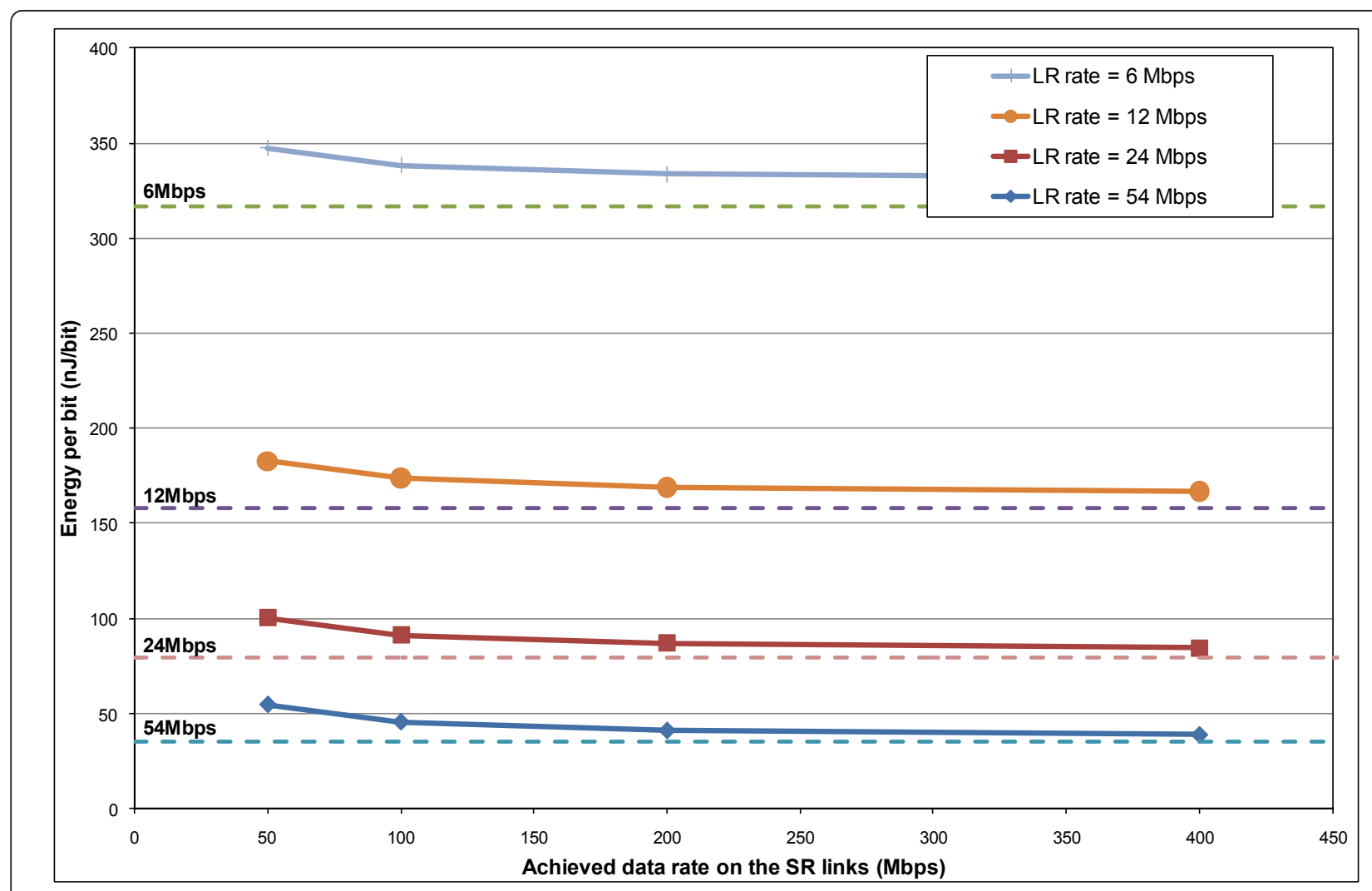

Figure 5 Energy efficiency in cooperation scenario (WiMedia-WiFi).

different plots for different achieved data rate on the LR from the cluster head $\left(\mathrm{MT}_{\mathrm{B}}\right)$ to the AP. It can be observed from the figure that the achieved data rate on the LR link has more effect on the energy efficiency than the data rate achieved on the SR. This can be explained by the higher energy consumption in the LR interface (IEEE802.11g) compared to the SR interface (WiMedia), as well as the higher data rates achieved on the SR links. MTs spend longer time sending the same amount of data on the WLAN interface with higher energy consumption rate. The horizontal lines represent the energy efficiency of the WLAN IEEE802.11 at different data rates. This figure can be used to evaluate the worth of using cooperation based on the data rates achieved on the cooperation links and the data rate achieved on the direct connection without using cooperation.

To elaborate more, looking at Figure 5, we can conclude that it is not energy profitable to use cooperation, if the LR direct link between the source MT with data and the AP can achieve $54 \mathrm{Mbps}$ (highest data rate). In this case, cooperation will always cost more energy to deliver the data to the AP; no matter what rates can be achieved on the SR and the LR links of the cooperation scenario. On the other hand, it is usually rewarding to use cooperation, if the LR direct link can at most achieve $6 \mathrm{Mbps}$, especially if high data rates can be achieved on the cooperation links.

Figure 5 shows the energy efficiency (consumed energy per bit), which is a good indication of the possible energy gains, but do not provide quantitative enhancement. In Figure 6, we show the energy enhancement compared to the direct communication with no cooperation. Different cases representing different achieved data rates on the direct LR link, as well as on the SR and LR links of the cooperation scenario.

The results in Figure 6 confirm the previous observation, which states that the achieved data rate on the LR link of the cooperation scenario has more effect on the energy savings than the data rate on the SR link. It can be seen that high energy savings can be achieved, reaching more than $80 \%$, when the direct LR link can only achieve $6 \mathrm{Mbps}$, while the LR link in the cooperation scenario can achieve high data rate of $54 \mathrm{Mbps}$. This is rather a theoretical limit than practical one, since the channel conditions from the $\mathrm{MT}_{\mathrm{B}}$ to $\mathrm{AP}$ may be hard to achieve that much gain compared to the channel conditions between $\mathrm{MT}_{\mathrm{A}}$ and the $\mathrm{AP} . \mathrm{MT}_{\mathrm{A}}$ and $\mathrm{MT}_{\mathrm{B}}$ have to be in the vicinity of each other to establish an SR 


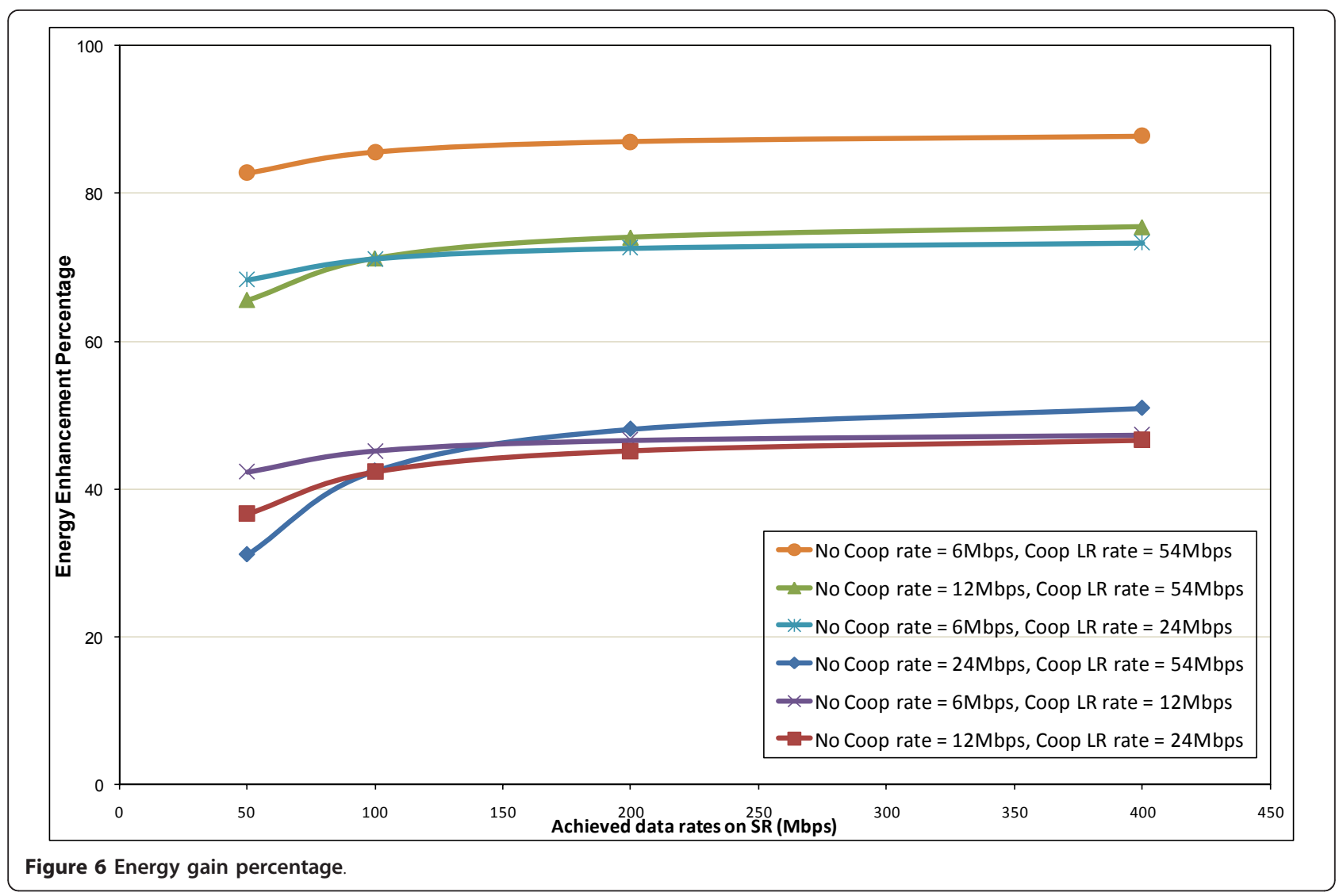

communication using WiMedia, especially with the very high data rates.

The above statement leads to our next set of results. Based on the fact that the channels between $\mathrm{MT}_{\mathrm{A}}$ and $\mathrm{AP}$ and $\mathrm{MT}_{\mathrm{B}}$ and $\mathrm{AP}$ may be hard to be that much different, $\mathrm{MT}_{\mathrm{B}}$ may choose to use more MTs in the middle to reach the AP. This scenario is envisioned in Figure 3, where the cluster head $\mathrm{MT}_{\mathrm{B}}$ uses $K$ hops to relay the data to the BS/AP. This case is represented with the more general Equation (7). We use Equation (7) to calculate the energy efficiency when using multiple relays on the route from source to AP, which is shown in Figure 7. The energy efficiency is plotted versus the number of relays between the cluster head and the AP (parameter $K$ in the equations). The horizontal lines in the figure again represent the energy efficiency of the WLAN IEEE802.11 at different data rates. It can clearly be seen that the energy consumption increases with the increase of number of hops. The interesting observation is the effect of the data rate of the SR links on the increase in energy consumption. It can be observed that the increase in energy consumption with the addition of relays is more significant when the data rate of the SR links is low. This indicates that more hops can be tolerated when higher data rates can be achieved on the SR links. This can clearly be seen in the case when the SR link rate is $50 \mathrm{Mbps}$ and the LR link rate is $54 \mathrm{Mbps}$. In this case, we can still achieve energy gain compared to the LR direct link with $24 \mathrm{Mbps}$, even with six hops between the cluster head and the AP. This observation is very important in verifying the concept of the SR cooperation. Achieving high data rates on the SR link probably implies shorter distance between communicating nodes, which may lead to the necessity of more hops to reach an MT with good channel to the AP, which can provide the high data rate assumed (54 Mbps). The results in Figure 7 can be used as guidelines to determine the limit on the number of hops allowed in the cooperation scenario, based on the data rate of the direct LR link and the data rates of the SR links and LR link of the cooperation. As an example, we assume that the direct link between an MT and the WLAN AP can achieve data rate of $24 \mathrm{Mbps}$. If we look at the graph with SR rate of $100 \mathrm{Mbps}$ and LR rate of 54 Mbps, the MT saves energy using cooperation as long as it can reach, in three 100-Mbps SR hops or less, an MT with a good channel providing $54 \mathrm{Mbps}$ link to the AP.

Again, we plot the percentage of energy gains for some interesting cases in Figure 8. The different plots 


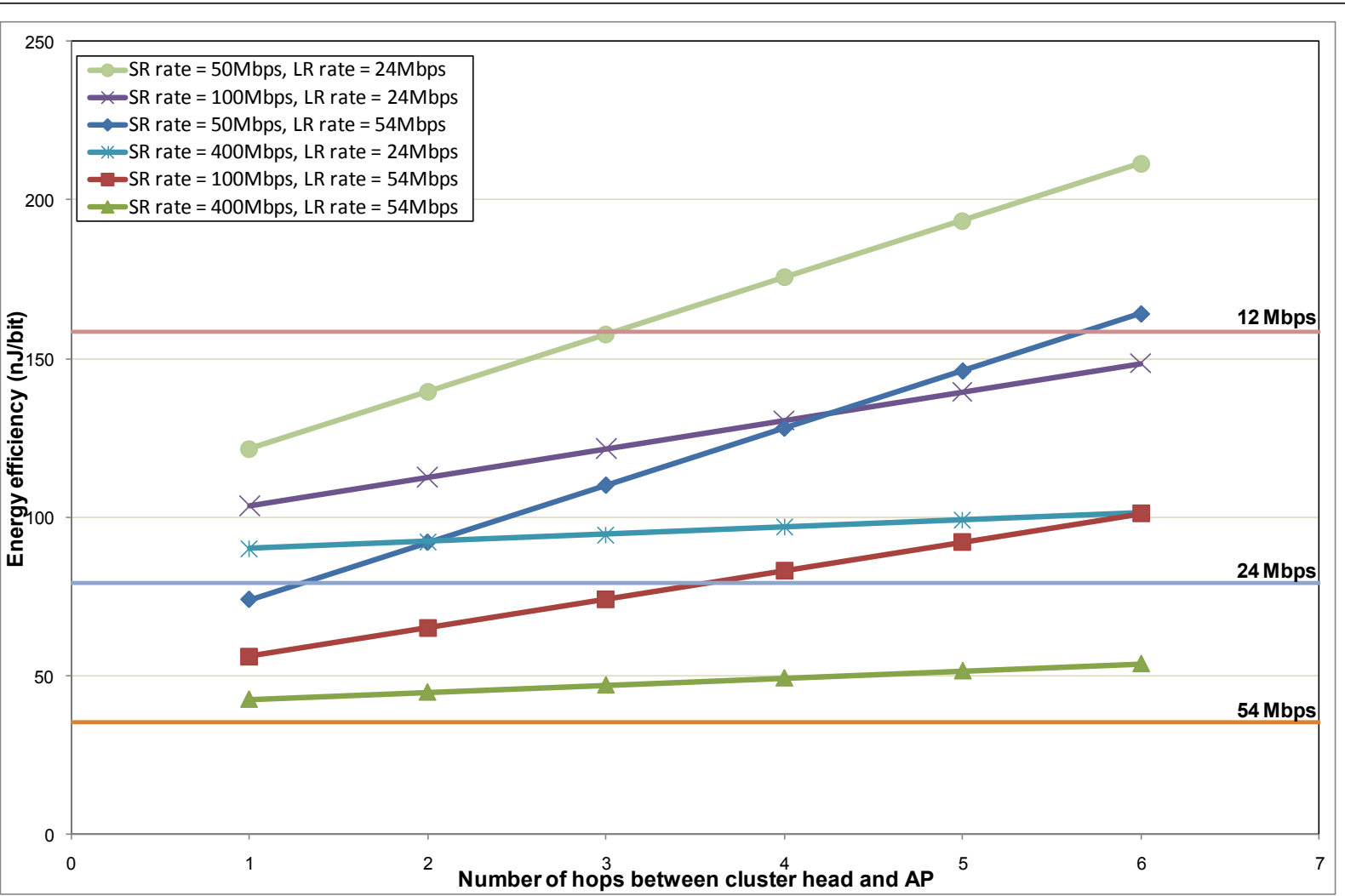

Figure 7 Energy efficiency ( $\mathrm{J} / \mathrm{bit}$ ) versus the number of relays.

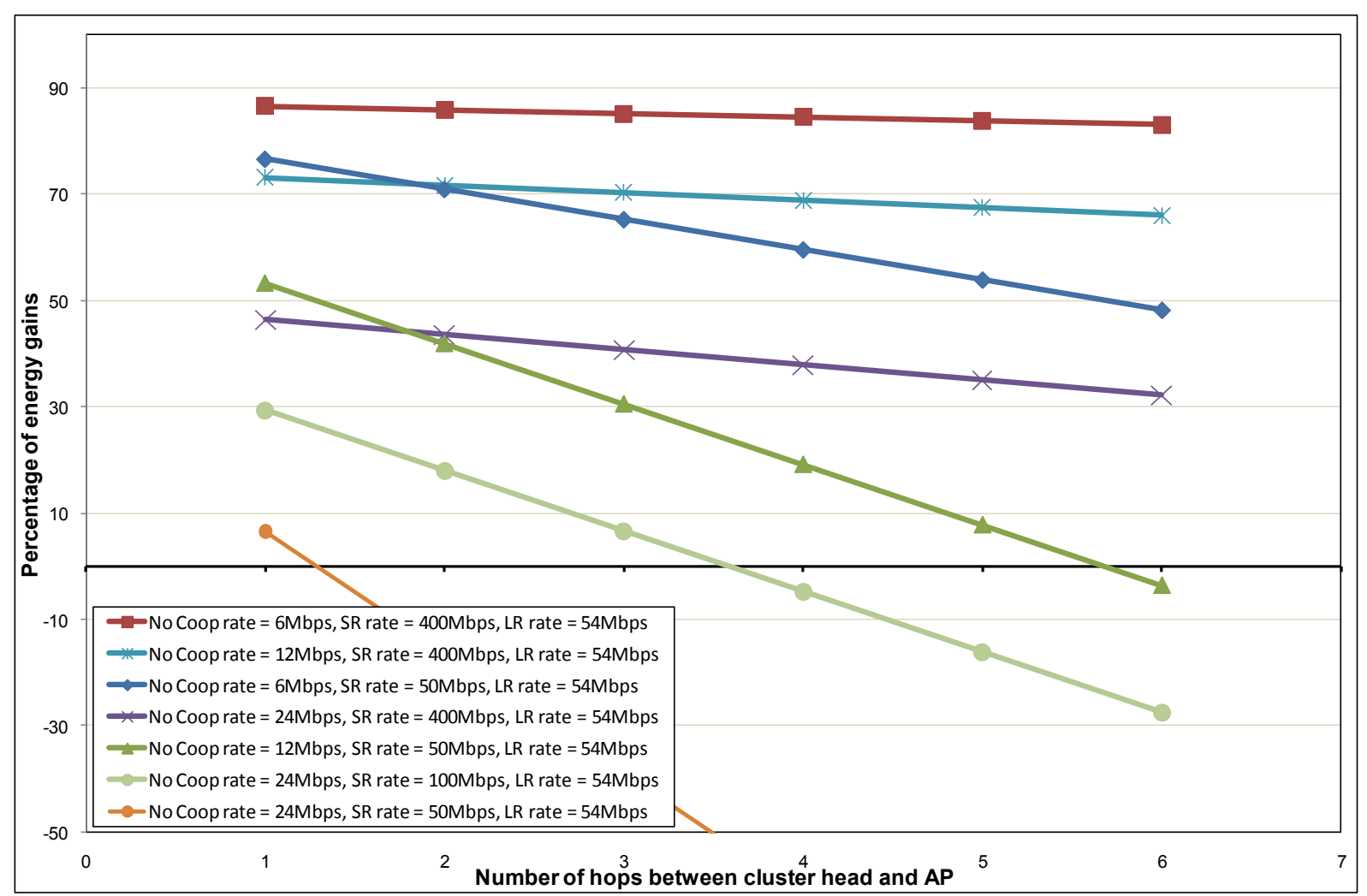

Figure 8 Percentage of energy gain. 
present different comparison cases, where we show the percentage of energy gain for different achieved data rates on the SR and LR of the cooperation links compared to different condition of the direct LR channel between the source MT sending data and the LR AP. It can be seen that in this figure, we show the negative values of the percentage. This is done to show the limit on the number of hops between the cluster head and the AP. For example, if the direct link can achieve 24 Mbps, a maximum of one hop should be allowed to achieve energy gains, if the SR link rate is 50 Mbps and the LR rate of the last hope in the cooperation is 54 Mbps. On the other hand, up to three hops can still achieve energy gain if the SR links can achieve 100 Mbps and the LR link can achieve 54 Mbps. Moreover, high energy savings can be achieved compared to a direct LR link (No cooperation) of $6 \mathrm{Mbps}$ data rate, even with six relaying hops, as long as $54 \mathrm{Mbps}$ is achieved on the LR link of the cooperation scenario.

From the results shown in this section, we can quantify the energy gains that can be achieved using our proposed cooperation approach. We showed that high energy gains can be achieved, but the exact value of the gain depends mainly on the achieved data rates on the different links. The cooperation approach hence tries to use SR relaying to reach an MT with good channel to the AP.

We showed that $50 \%$ enhancement in energy consumption and higher can be achieved if one hop is enough to reach an MT with good channel to the AP. In addition, we showed that even with multiple hops, cooperation can still achieve energy savings as long as the data rates achieved in the cooperation case is much higher than the data rate of the direct link.

It has to be noted here that these gains are calculated on the assumption of perfect operation of the cooperative algorithms. The practical achieved gains will be lower due to overheads and imperfections. For example, the MAC layer should add more energy consumption, especially in a dense environment with many users contending for channels. In addition, the overhead of performing the cooperation also decreases the energy gains. Some of the cooperation overheads are node discovery, cluster formation and cooperation negotiations. Within the course of our research efforts, we plan to optimize these functionalities to minimize their effects on energy gains.

\subsection{WiFi $(802.11 \mathrm{~g})$ as both the $L R$ and $S R$}

In this use case, we consider WiFi $802.11 \mathrm{~g}$ as the LR technology of choice as well as the SR technology used for cooperative communications between MTs. From the table, it can be seen that the power dissipation during the transmission state is the highest. The power consumption during the receiving is not much lower than that of the transmission state. The power dissipation during the sleep state is much lower than all other states. From the table, it can be calculated that the power consumption during the sleep state is $3 \%$ of that during transmission state. Hence, our approach tries to keep the WiFi interface in the sleep state as long as possible to save energy.

For IEEE $802.11 \mathrm{~g}$, different data rates can be achieved based on the channel between the transmitter and the receiver. We already showed the effects of different conditions of channels in the previous use case (combination of WiFi and WiMedia) in Figure 5. In this use case, we show the range of energy gains that can be achieved compared to different data rates of the direct LR link to the AP. In Figure 9, we show the percentage of energy gains that can be achieved with different data rates achieved on the direct LR link, and the cooperation SR and LR links. The plots show the effect of varying the data rate on the SR or the LR of the cooperation scenario. The dotted graphs assume that the SR links can achieve the highest data rate of $54 \mathrm{Mbps}$ and show the effect of varying the data rates on the last LR hop of the cooperation scenario. On the other hand, the solid graphs examine the effect of the variation in the data rates on the SR links, while the last LR hop always achieves a data rate of 54 Mbps. As can be seen from the figure, the data rates on SR links have more effect on the achieved energy savings. This can be explained by the idea that in the SR link the transmitter and the receiver are MTs, while in the LR link the receiver is the AP, which is not included in the calculations.

In Figure 10, the percentage of energy gains is plotted against the number of relays between the cluster head and the LR AP. In the results shown, the direct LR link is assumed to achieve a low data rate of $6 \mathrm{Mbps}$. Different data rates are assumed on the SR and LR of the cooperation scenario. From the plots in the figure, it can be seen that no energy gain can be achieved with more than three hops, even with the highest data rates possible on the WLAN (54 Mbps) achieved on both the SR and LR links of the cooperation. This shows the effect of the ratio between the achieved data rates on the SR and the LR technologies. In the previous use case (WiFi and WiMedia), more hops are possible since the ratio of possible achieved data rates is much higher than the current case (WiFi as LR and SR). This emphasizes the importance of using an SR technology with high data rates, like WiMedia, even with the limit on its coverage range, since the limit on the number of hops is more loose.

From the results in this use case, it can be concluded that energy savings can still be achieved when using IEEE802.11g as both the SR and LR technologies, but 


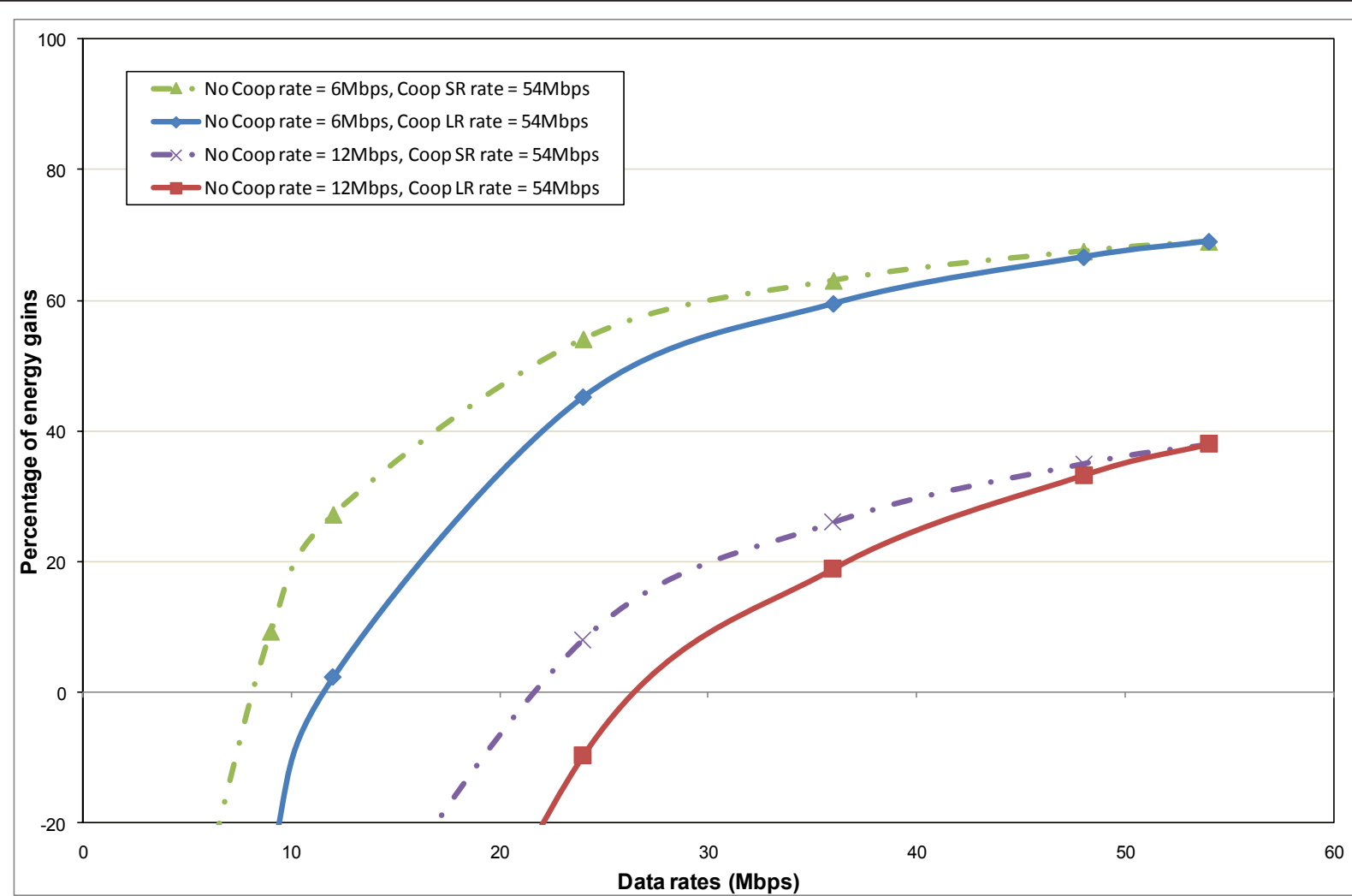

Figure 9 Percentage of energy gains with different data rates

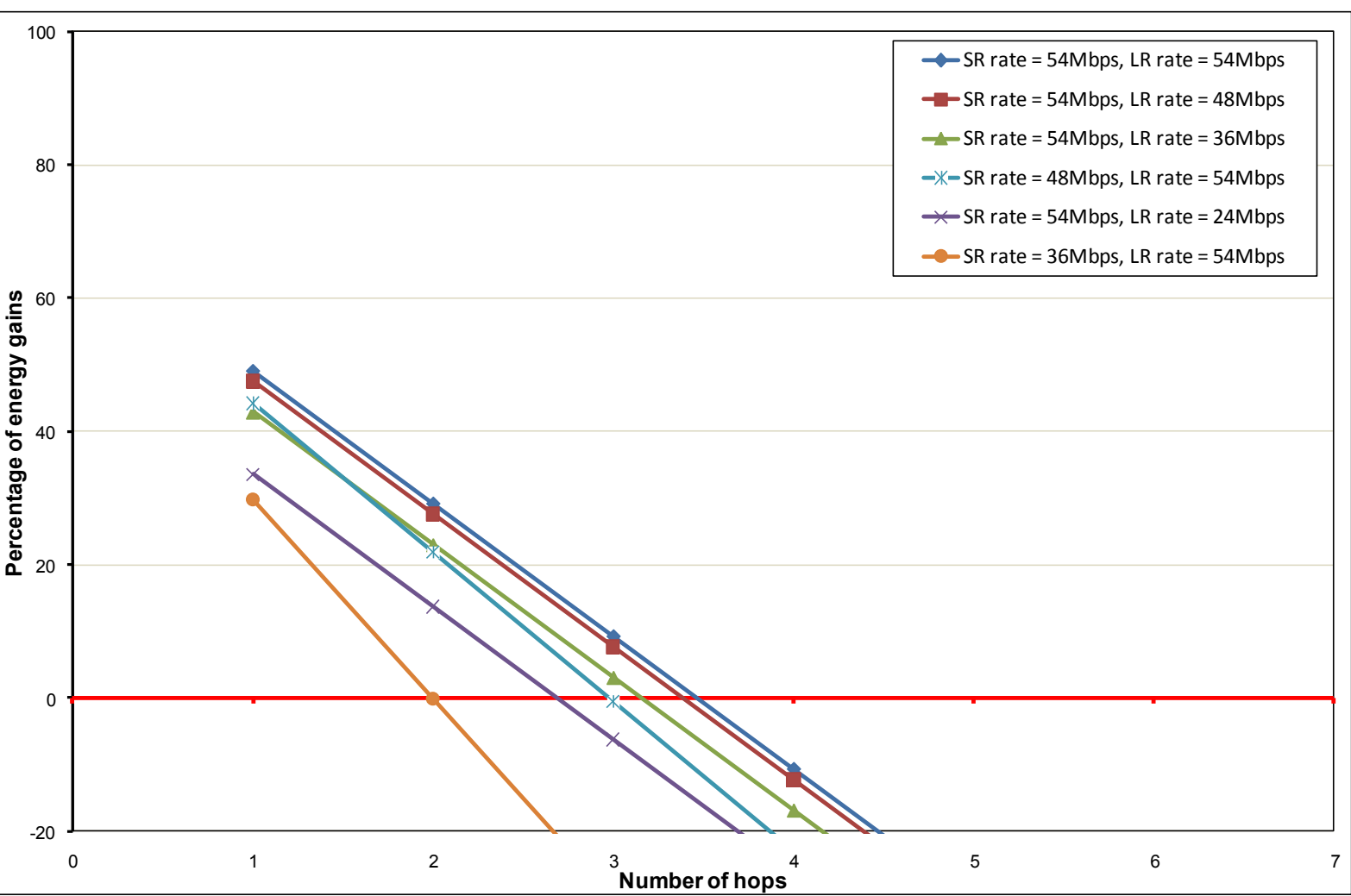

Figure 10 Percentage of energy gains versus number of hops 
the gains are much lower than the case of WiMedia and IEEE802.11g. In the best case scenario, using IEEE802.11g as SR and LR, the maximum achievable energy saving is $69 \%$ when having $54 \mathrm{Mbps}$ on all the links in the cooperation case compared to $6 \mathrm{Mbps}$ data rate on the direct LR link.

\subsection{WiMAX as LR and WiFi (802.11g) as SR}

In the last use case, the SR technology is assumed to be IEEE802.11g and the LR technology is WiMAX. In this use case, we will just show the percentage of the energy gains in the best case scenario, since the arguments for different scenarios are similar to previous use cases. We assume that the direct LR link to the WiMAX BS is suffering from bad conditions and hence can only achieve low data rate of $5.33 \mathrm{Mbps}$ as an exemplary value of low data rates of WiMAX. We also assume that the last LR hop to the WiMAX BS in the cooperation case can achieve the high data rate of $64 \mathrm{Mbps}$, the highest data rate achieved on WiMAX. According to these assumptions, we plot the percentage of achieved energy savings against the number of relays between the cluster head and the WiMAX BS in Figure 11.

From the results in the figure, it can be seen that 50\% is the maximum energy enhancement that can be achieved in this use case of using WiMAX as the LR technology and IEEE802.11g as the SR technology. It can also be observed that only a maximum of three hops is possible to achieve energy gains. More than three hops in this use case result in consuming more energy than the direct LR link. The reason is again the lower ratio of possible data rates on the IEEE802.11g and on the WiMAX compared to the ratio in the first use case of WiMedia and IEEE802.11g.

Based on the discussion and numerical results in this section, it is observed that energy savings can be achieved using cooperative communication by combining SR technology with LR technology. It was shown that at least $50 \%$ energy savings can be achieved in all considered use cases, with even higher energy savings (reaching almost $80 \%$ ) in the case of WiMedia with WLAN. The achieved energy gains basically depend on the ratio between the achieved data rate on the links of the cooperation compared to the possible data rate on the direct LR link. It has to be noted that these gains are performance targets, which will be hard to achieve in real demonstration scenario due to overheads and imperfection of wireless channels. The study in this article presents a quantification of the possible energy savings when using cooperation between MTs to relay data

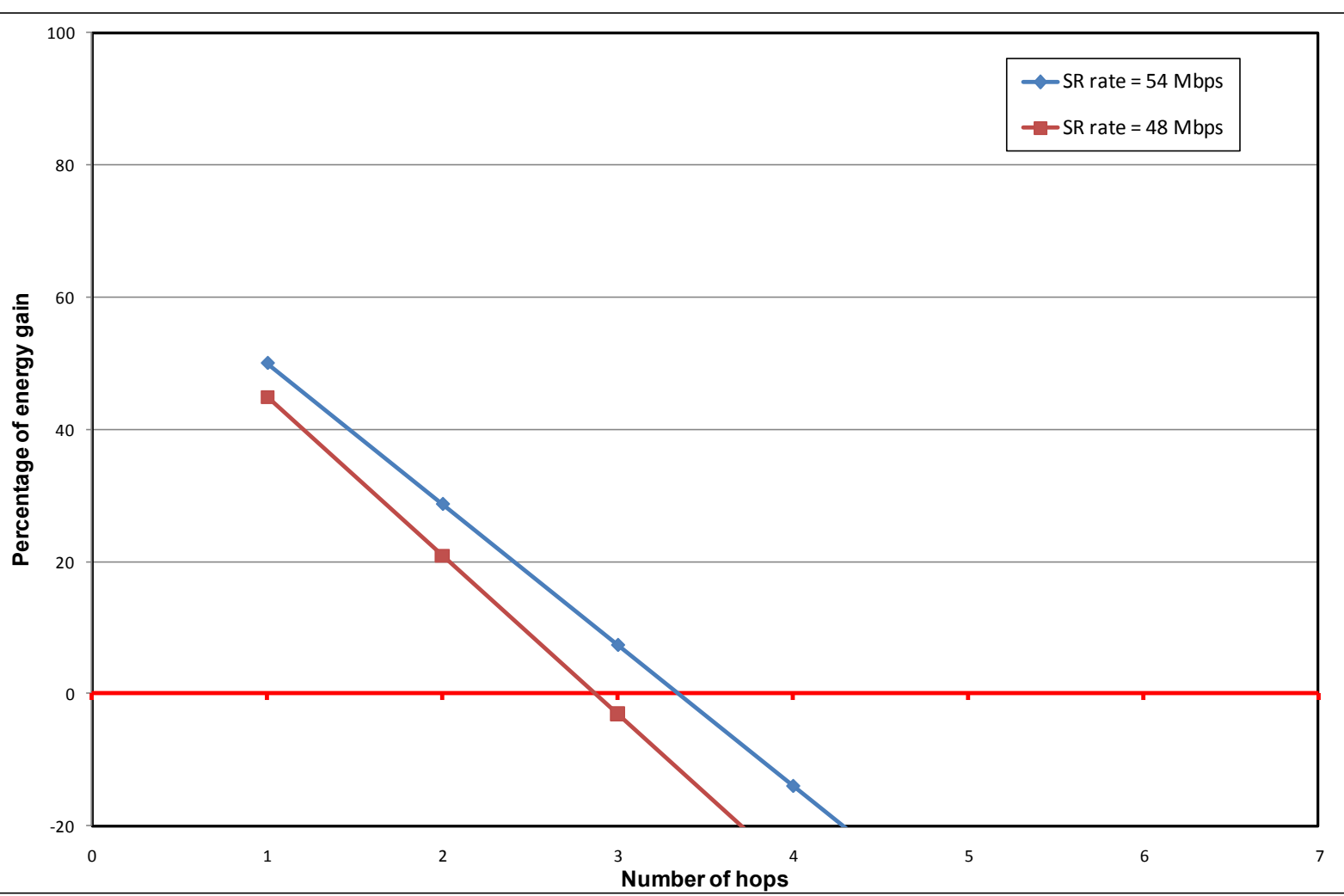

Figure 11 Percentage of energy gains versus the number of hops 
from MTs experiencing bad channels towards the BS. The formulas derived can be used to determine whether cooperation will be useful based on the characteristics of the considered scenario.

\section{Conclusion}

Wireless mobile networks are advancing with respect to the achieved data rates and availability. Mobile users can now enjoy multiple advanced multimedia applications on their devices while on the move. These advancements are accompanied by an increase in the power requirements of the current multiple interface mobile devices. The problem is that the battery industry is not advancing with the same pace and there is a growing gap between what can be achieved by the battery industry and the energy requirements of the energy hungry advanced applications and multi-standard mobile devices. Without any new approaches for energy saving, 4G mobile users will relentlessly be searching for power outlets rather than network access and becoming once again bound to a single location.

To avoid the 4G "energy trap", we propose to use SR cooperation among MTs to save energy by taking advantage of the good channel conditions of the SR links. This article provides quantitative analysis of the proposed cooperative solutions to save energy in multistandard mobile devices. The article derives formulas to calculate energy consumption in the non-cooperative and cooperative cases. The derivation takes into account the energy consumed in the original source nodes as well as the relaying nodes. The numerical results show the potential energy savings in the MTs in different use cases, taking into consideration variable channel conditions. We illustrate that up to $80 \%$ energy savings can be achieved, if WiMedia is used as the cooperative SR technology. The energy savings vary depending on the technologies used for the SR and LR communications and the achieved data rates based on the channel conditions, but all cases achieve at least 50\% energy savings. The results also provide a limit on the maximum number of relays that can be used in different cases. This article can be considered as a reference to determine whether cooperation would be beneficial based on the evaluated scenario-available technologies and channel conditions among mobile devices and between mobile devices and the access points. We also show that even if global energy saving in the network cannot be achieved, some mobile devices can benefit from SR cooperation by moving the burden of energy consumption to other mobile devices (relays) in exchange of some kind of payments. This concept opens a new topic for business models involving mobile users and service providers. This is the subject of some of our future research directions, along with efforts towards the optimization of functionalities, like node discovery, cluster formation and cooperation negotiations, to minimize their overhead effects on energy gains.

\section{Acknowledgements}

This study has been performed in the framework of the ICT project ICT248577 C2POWER, which is funded by the European Union.

\section{Competing interests}

The authors declare that they have no competing interests.

Received: 2 October 2011 Accepted: 2 May 2012 Published: 2 May 2012

\section{References}

1. F Fitzek, M Katz, Cognitive Wireless Networks: Concepts, Methodologies and Visions Inspiring the Age of Enlightenment of Wireless Communications, (Springer, Berlin, 2006)

2. J Rodriguez, P Marques, A Radwan, K Moessner, R Tafazolli, M Raspopoulos, S Stavrou, P Trapps, D Noguet, K Sithamparanathan, A Gomes, R Piesiewick, H Mokrani, A Foglar, C Verikoukis, Cognitive radio and cooperative strategies for power saving in multi-standard wireless devices, in Future Network and Mobile Summit 2010, Florence, Italy, pp. 1-8 (June 2010)

3. FHP Fitzek, MD Katz, in Cognitive Wireless Networks Concepts: Methodologies and Visions Inspiring the Age of Enlightenment of Wireless Communications, (Springer, Berlin, 2007)

4. B Smith, Bluetooth umbrella covers low-Power Wibree. WirelessWeek http:// www.wirelessweek.com/article.aspx?id=149088 (June 2007)

5. ECMA-368: High Rate Ultra Wideband PHY and MAC Standard, 3 (2008)

6. A Muqattash, G Heidari-Bateni, Dynamic power save modes. (2007) US 2007/0160027 A1

7. Y Chen, N Smavatkul, S Emeott, Power management for VolP over IEEE 802.11 WLAN, in Proc IEEE Wireless Communications and Networking Conference (WCNC), Atlanta, Georgia, USA, pp. 1648-1653 (March 2004)

8. WMMTM Power Save for Mobile and Portable Wi-Fi ${ }^{\mathbb{R}}$ CERTIFIED Devices. WiFi Alliance (December 2005)

9. P Gong, P Xue, DK Kim, Enhancing the performance of UWB-based WPANs using cooperative PNC relaying. IEICE Trans Commun. E91-B(9), 3000-3003 (2008)

10. F Fitzek, M Pedersen, GP Perrucci, T Larsen, Energy and link measurements for mobile phones using IEEE802.11 b/g, in Proc International ICST Workshop on Wireless Networks Measurement (WiNMEE 2008), Berlin, Germany, (2008)

11. F Fitzek, J Heide, MV Pedersen, G Ertli, M Katz, Multi-hop versus overlay networks: a realistic comparison based on energy requirements and latency, in Proc IEEE Vehicular Technology Conference (VTC spring), Budapest, Hungary, pp. 1-5 (May 2011)

12. J Alonso-Zárate, E Kartsakli, C Verikoukis, L Alonso, Persistent RCSMA: a MAC protocol for a distributed cooperative ARQ scheme in wireless networks. EURASIP J Adv Signal Process (Special Issue on Wireless Cooperative Networks) 817401 (2008). 13 (2008)

13. J Alonso-Zarate, L Alonso, C Verikoukis, Performance analysis of a persistent relay carrier sensing multiple access protocol. IEEE Trans Wirel Commun. 8(12), 5827-5831 (2009)

14. G Shor, How Bluetooth, UWB, and 802.11 stack up on power consumption. http://www.wirelessnetdesignline.com/howto/207200448; jsessionid=FGA0FYAOTYLA5QE1GHOSKHWATMY32JVN?pgno=1 15. UM230 WiMAX USB modem data sheet

doi:10.1186/1687-1499-2012-159

Cite this article as: Radwan and Rodriguez: Energy saving in multistandard mobile terminals through short-range cooperation. EURASIP Journal on Wireless Communications and Networking 2012 2012:159. 